\title{
Prompt Photons with Associated Jets in Photoproduction at HERA
}

\author{
S. Chekanov \\ For the ZEUS Collaboration \\ DESY Laboratory, 22607, Hamburg, Germany. \\ On leave from the HEP division, Argonne National Laboratory, \\ 9700 S.Cass Avenue, Argonne, IL 60439, USA \\ E-mail: chekanov@mail.desy.de
}

\begin{abstract}
Prompt photons, together with an accompanying jet, have been studied in the photoproduction regime of $e p$ scattering measured with the ZEUS detector at HERA. Predictions based on leading-logarithm parton-shower Monte Carlo models and nextto-leading-order (NLO) QCD underestimate the $\gamma+$ jet cross sections for transverse energies of prompt photons below $7 \mathrm{GeV}$, while the $k_{T}$-factorisation QCD calculation agrees with the data in this region.
\end{abstract}

\section{Theoretical calculations}

Events with an isolated photon (prompt photon) are important tool to study hard interaction processes since such photons emerge without the hadronisation phase. In particular, final states with a prompt photon together with a jet are directly sensitive to the quark content of the proton through the elastic scattering of a photon by a quark, $\gamma q \rightarrow \gamma q$ (see Fig. 1). However, QCD contributions to this lowest-order process lead to a significant sensitivity to the gluon structure function. In particular, a contribution to prompt-photon events from $g q \rightarrow q \gamma$ process, in which the photon displays a hadronic structure (resolved process), is important [2-4]. Thus, prompt-photon events can constrain both proton and photon parton densities. In addition, a number of QCD predictions [2-5] can be confronted with the data.

The next-to-leading order (NLO) calculations based on the collinear factorisation and the DGLAP formalism were performed by Krawczyk and Zembrzuski (KZ) [3] and by Fontanaz, Guillet and Heinrich (FGH) [4]. No intrinsic transverse momentum of the initial-state partons in the proton was assumed. The renormalisation scale for such calculations was taken to be $\mu_{R}=E_{T}^{\gamma}$. In case of the KZ predictions, the GRV parameterisation of the proton, photon and fragmentation function were used $[6,7]$. For the FGH calculations, MRST01 proton structure function and the AFG02 structure function for the photon were used [7]. The FGH NLO calculation takes into account high-order terms in the QCD expansion which have not been considered in the $\mathrm{KZ}$ approach.

The QCD calculations based on the $k_{T}$-factorisation [8] approach were performed by A. Lipatov and N. Zotov (LZ) [5]. The unintegrated quark and gluon densities of the proton and photon using the Kimber-Martin-Ryskin (KMR) prescription [9] were used. As for the NLO QCD, both direct and resolved contributions are taken into account. 
For all the calculations discussed above, an isolation requirement $E_{T}^{\gamma}>0.9 E_{T}^{\text {tot }}$ was used, where $E_{T}$ is the transverse energy of the photon and $E_{T}^{\text {tot }}$ is the total energy of the photon-candidate jet reconstructed with the longitudinally-invariant $k_{T}$ algorithm in inclusive mode [10]. The $\gamma+$ jet cross sections were corrected for hadronisation effects using a Monte Carlo (MC) simulation.

\section{Event reconstruction}

Each $k_{T}$ jet, reconstructed from energy-flow objects (EFO), was classified as either a photon candidate or a hadronic jet. The photon-candidate jet was required to consist of EFOs without associated tracks and to be within the central tracking detector, $-0.74<\eta^{\gamma}<1.1$. For this jet, $E_{\mathrm{EMC}} / E_{\mathrm{tot}}>0.9$ is required, where $E_{\mathrm{EMC}}$ is the energy reconstructed in the electromagnetic part of the CAL and $E_{\text {tot }}$ is the total energy of this jet. After correction for energy losses, the cut $E_{T}^{\gamma}>5 \mathrm{GeV}$ was applied.

ZEUS
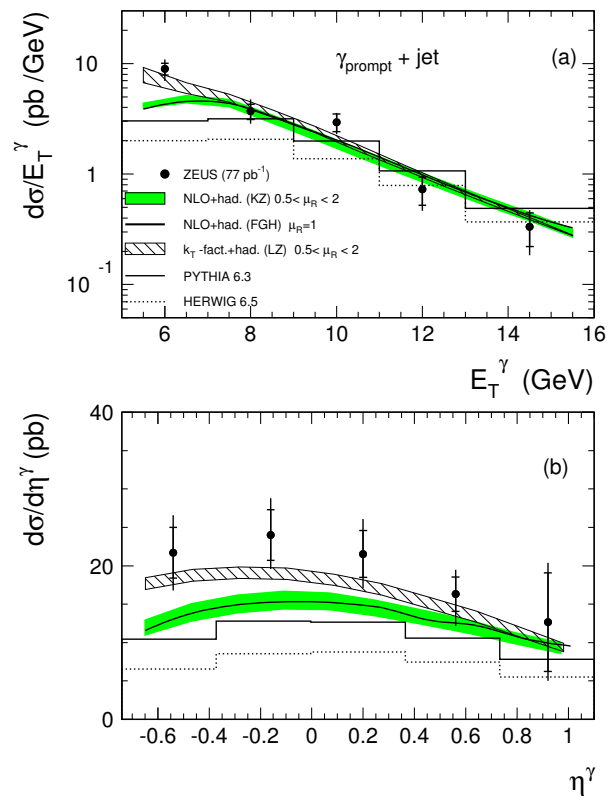

ZEUS
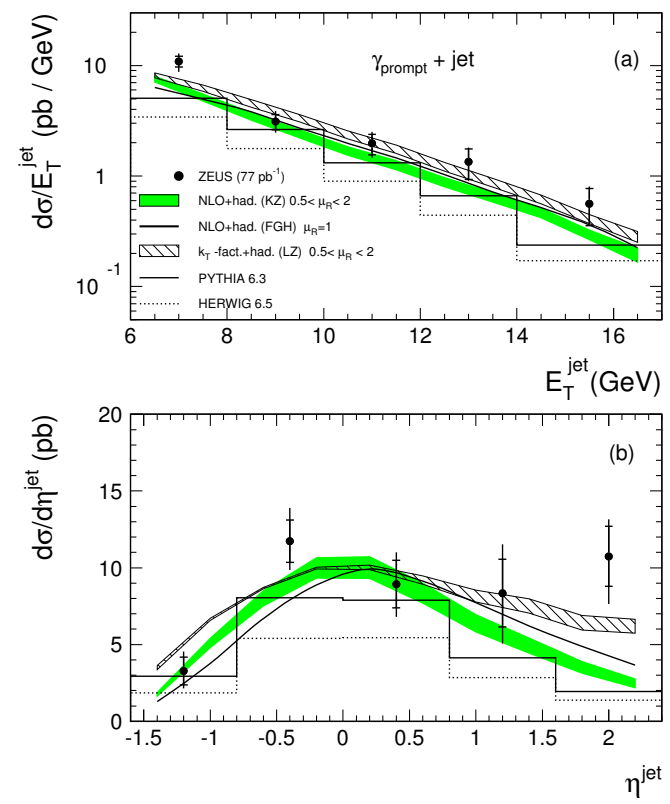

Figure 1. The differential $\gamma+$ jet cross sections as functions of $E_{T}$ and $\eta$ for the prompt photon and the jet. The data are compared to QCD calculations and MC models. The shaded bands correspond to a typical renormalisation scale uncertainty which was obtained by changing $\mu_{R}$ by a factor of 0.5 and 2 . 

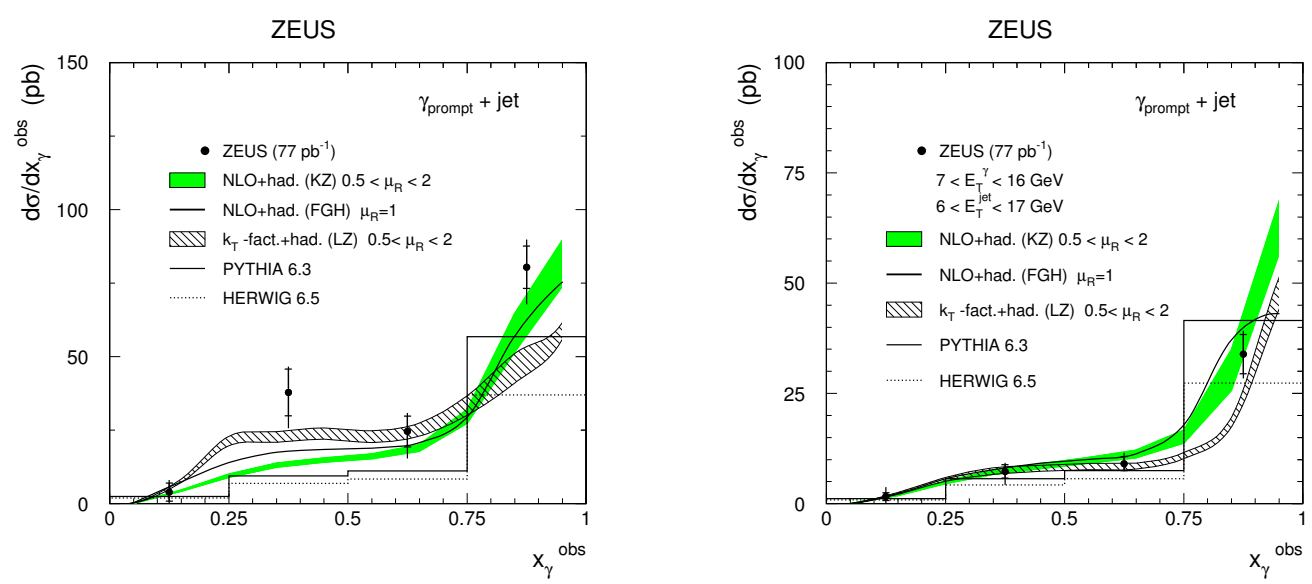

Figure 2. The $x_{\gamma}^{\text {obs }}$ cross section for $\gamma+$ jet events compared to the NLO QCD calculations and MC models for $E_{T}^{\gamma}>5 \mathrm{GeV}$ (left) and $E_{T}^{\gamma}>7 \mathrm{GeV}$ (right).

Hadronic jets, after correction for energy losses, were selected in the kinematic range $E_{T}^{\mathrm{jet}}>6 \mathrm{GeV},-1.6<\eta^{\mathrm{jet}}<2.4$. If more than one jet was found within the above kinematic cuts, the jet with the highest $E_{T}^{\text {jet }}$ was accepted.

For the prompt-photon identification, the conversion-probability method was used [11]. In contrast to the shower-profile approach adopted in previous HERA measurements, the present approach uses the probability of conversion of photons to $e^{+} e^{-}$pairs in detector elements and inactive material (mainly the ZEUS superconducting coil) in front of the barrel calorimeter (BCAL). Since the conversion probability for a single photon is smaller than for multiphoton events arising from neutral meson decays $\left(\pi^{0}, \eta\right.$, etc. $)$, one can extract the $\gamma$ signal by performing a statistical background subtraction.

To determine the number of charged particles in photon shower, the ZEUS barrel preshower detector (BPRE) [12] located in front of the BCAL was used. The measured output, calibrated in minimum ionising particle units (mips), is proportional to the energy loss of the incident particle after interaction with inactive material. The response of the BPRE to single isolated photons was verified using deeply virtual Compton scattering events. For the $\gamma+$ jet, the BPRE signal for the $\gamma$ candidates was fitted using a MC model with and without prompt photons, and the number of events associated with the photon signal was extracted.

\section{$3 \quad$ Results and conclusions}

The total cross section for the process $e p \rightarrow e+\gamma_{\text {prompt }}+$ jet $+X$ for $0.2<y<0.8$, $Q^{2}<1 \mathrm{GeV}^{2}, 5<E_{T}^{\gamma}<16 \mathrm{GeV}, 6<E_{T}^{\mathrm{jet}}<17 \mathrm{GeV},-0.74<\eta^{\gamma}<1.1,-1.6<$ $\eta^{\text {jet }}<2.4$ and $E_{T}^{\gamma \text {,(true })}>0.9 E_{T}^{\gamma}$ was measured to be $\sigma\left(e p \rightarrow e+\gamma_{\text {prompt }}+\right.$ jet $\left.+X\right)=$ $33.1 \pm 3.0$ (stat.) ${ }_{-4.2}^{+4.6}$ (syst.) pb.

This value agrees well with the LZ $k_{T}$-factorisation calculations $\left(30.7_{-2.7}^{+3.2} \mathrm{pb}\right)$, but higher than for the NLO QCD $\left(23.3_{-1.7}^{+1.9} \mathrm{pb}(\mathrm{KZ})\right.$ and $\left.23.5_{-1.6}^{+1.7} \mathrm{pb}(\mathrm{FGH})\right)$ and MC models.

The differential cross sections as functions of $E_{T}$ and $\eta$ for the prompt-photon candidates and for the accompanying jets are shown in Figure 1. The MC differential cross sections do 
not rise as steeply at low $E_{T}^{\gamma}$ as do the data. The KZ NLO prediction describes the data better. However, it underestimates the observed cross section at low $E_{T}^{\gamma}$ and in the forward jet region. The FGH prediction is similar to the KZ NLO. The LZ prediction based on the $k_{T}$-factorisation approach gives the best description of the $E_{T}$ and $\eta$ cross sections.

Figure 2(left) shows the distribution for $x_{\gamma}^{\text {obs }}$ defined as $\sum_{\gamma, \text { jet }}\left(E_{i}-P_{Z}^{i}\right) /\left(2 E_{e} y\right)$ (the sum runs over the photon candidate and the hadronic jet). The difference between the NLO QCD and the data is mainly concentrated in the resolved region.

It is important to verify the level of agreement with NLO when the minimum transverse energy of the detected prompt photons is increased from $5 \mathrm{GeV}$ to $7 \mathrm{GeV}$. In comparison with previous measurements, such a choice may emphasize different aspect of contributions of high-order QCD radiation, since the transverse energy of the prompt-photon is larger than that of the jet.

Figure 2(right) shows the corresponding $x_{\gamma}^{\text {obs }}$ distribution. For the $E_{T}^{\gamma}>7 \mathrm{GeV}$ cut, both the NLO QCD and the LZ predictions agree well with the data. There is also good agreement for the $E_{T}$ and $\eta$ kinematic variables [11].

Acknowledgements. I thank M. Fontannaz, G. Heinrich, M. Krawczyk, A. Lipatov, N. Zotov and A. Zembrzuski for discussions and for providing the QCD calculations.

\section{References}

[1] Slides: http://indico. cern. ch/contributionDisplay . py? contribId=219\&sessionId=6\&conf Id=9499 .

[2] L. Gordon, W. Vogelsang, Phys. Rev. D52, 58 (1995).

[3] M. Krawczyk, A. Zembrzuski, Phys. Rev. D64, 14017 (2001).

[4] M. Fontannaz, J. P. Guillet, G. Heinrich, Eur. Phys. J. C21, 303 (2001).

[5] A. Lipatov, N. Zotov, Phys. Rev. D72, 054002 (2005).

[6] M. Glück, E. Reya, A. Vogt, Z. Phys. C67, 433 (1995); M. Glück, E. Reya, A. Vogt, Phys. Rev. D45, 3986 (1992).

[7] A. D. Martin, et al., Eur. Phys. J. C14, 133 (2000); P. Aurenche, J. P. Guillet, M. Fontannaz, Z. Phys. C64, 621 (1994).

[8] E. M. Levin, et al., Sov. J. Nucl. Phys. 53, 657 (1991); S. Catani, M. Ciafaloni, F. Hautmann, Nucl. Phys. B366, 135 (1991); J. Collins, R. Ellis, Nucl. Phys. B360, 3 (1991).

[9] M. A. Kimber, A. D. Martin, M. G. Ryskin, Phys. Rev. D63, 114027 (2001); G. Watt, A. D. Martin, M. G. Ryskin, Eur. Phys. J. C31, 73 (2003).

[10] S. Ellis, D. Soper, Phys. Rev. D48, 3160 (1993); S. Catani, et al., Nucl. Phys. B406, 187 (1993).

[11] ZEUS Collaboration, S. Chekanov, et al., Eur. Phys. J. C49, 511 (2007).

[12] S. Magill, S. Chekanov, Proceedings of the IX Int. Conference on Calorimetry (Annecy, Oct 9-14, 2000), B. Aubert, et al. (eds.), p. 625. Frascati Physics Series 21, Annecy, France (2001). 